\title{
Development of a biomarker for sucrose intake based on metabolomics
}

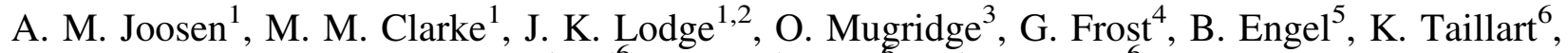 \\ A. J. Lloyd ${ }^{6}$, M. Beckmann ${ }^{6}$ and J. Draper ${ }^{6}$ \\ ${ }^{1}$ Cranfield University MK43 OAL, ${ }^{2}$ Northumbria University NE1 8ST, ${ }^{3}$ MRC-Human Nutrition Research CB1 9NL, \\ ${ }^{4}$ Imperial College London SW7 2AZ, ${ }^{5}$ University of Surrey GU2 7XH, ${ }^{6}$ Aberystwyth University SY23 $3 F G$, UK
}

Carbohydrates are the major source of energy in the diet and their intake is an important determinant of health. The intake of extrinsic sugars, such as sucrose, is of increasing public health concern as evidence suggests high intakes are associated with metabolic abnormalities leading to hyperlipidaemia, insulin resistance and increased risk of degenerative disease ${ }^{(1)}$. It is important, therefore, to have an accurate determination of dietary sucrose intake and metabolomics has recently been shown to be a useful tool for measuring dietary exposure $^{(2)}$. The aim of this study was to develop biomarkers of sucrose intake using metabolomics.

We performed a randomised, parallel, single-dose feeding study on 90 healthy females (aged $29.9 \pm 4.7 \mathrm{yr}, \mathrm{BMI} 23.3 \pm 2.5 \mathrm{~kg} / \mathrm{m}^{2}$ ) consuming either $0 \mathrm{~g}, 50 \mathrm{~g}$ or $100 \mathrm{~g}$ sucrose in $500 \mathrm{ml}$ water (30 subjects per group). Blood and urine samples were taken before and for $24 \mathrm{~h}$ post sucrose intake. Standard low carbohydrate meals were provided during the study period. Blood was analysed for markers of glucose control and lipid status, urine was measured for electrolytes and sucrose excretion. Urine and plasma samples underwent FIE-MS fingerprinting analysis using established protocols ${ }^{(3)}$, followed by GCMS and LCMS analysis.

FIE-MS fingerprinting analysis showed that the $3 \mathrm{~h}$ time point was the most informative in urine and plasma (PC-LDA $\mathrm{T}_{\mathrm{w}}$ values $>2$ ) and out of 120 explanatory signals, highlighted 12 metabolite signals in urine and 23 metabolite signals in plasma above a statistical significance threshold $\left(p<1 \times 10^{-4}\right)$ that were discriminatory and either positively or negatively correlated with sucrose intake over time. In urine, the main signals positively correlated with sucrose intake were attributed to sucrose at 3,6 and $9 \mathrm{~h}$, and fructose and erythronic acid at $3 \mathrm{~h}$. In plasma erythronic acid was also discriminatory and several unknowns were also positively correlated with sucrose intake. In urine and/or plasma samples several discriminatory signals attributed to keto- and hydroxy acids, medium to long chain non-esterified fatty acids and derivatives (acyl-carnitines), indicative of a fasting response, were highly abundant following consumption of water and were generally negatively correlated with sucrose intake, certain species increasing with time in the 0 g group (e.g acetylcarnitine, 3-OHbutanoic acid). GCMS and LCMS profiling analysis of urine and/or plasma samples confirmed fingerprinting data in that sucrose, fructose and erythronic acid were the strongest dose responsive discriminators for sucrose intake and metabolites associated with a fasting response being negatively correlated with sucrose intake.

In conclusion, we have found sucrose, fructose, and erythronic acid to be positively correlated with sucrose intake suggesting that these could be developed further into quantitative biomarkers of sucrose intake. A fasting-to-fed metabolic switch was also evident over time indicating that total energy intake during the study was low.

This work was supported by the Food Standards Agency (N05084) and the Department of Health (024/0044). Any views expressed are the author's own.

1. Malik VS, Popkin BM, Bray GA et al. (2010) Circulation 121, 1356-64.

2. Lloyd AJ, Fave G, Beckmann M et al. (2011) Am J Clin Nut 94, 981-91.

3. Fave G, Beckmann M, Lloyd AJ et al (2011) Metabolomics 7, 469-84. 